\title{
TWO NEW GENETIC MUTATIONS OF CADASIL DISEASE
}

Authors: O. López Agudelo ${ }^{1}$, L. Redondo Robles ${ }^{1}$, S. Martínez Peralta ${ }^{1}$, A.D. Murillo Hernández ${ }^{1}$, M.D. Calabria Gallego ${ }^{1}$, A. Sierra

Gómez¹, M. Álvarez Álvarez¹, J. Domínguez Díez¹, V.A. Vera Monge1 ${ }^{1}$ L. López Mesonero1, I. Gómez Estévez¹.

1 University Hospital of Salamanca, Neurology, Salamanca, Spain.

\section{BACKGROUND AND AIMS}

CADASIL disease is a genetic disease that causes microvascular dysfunction. More than $\mathbf{2 0 0}$ mutations have been described, especially involving cysteine residues.

We present two cases of patients with CADASIL disease due to two new different mutations in NOTCH 3 gene.

\section{METHOD}

Case 1: 73-year-old female admitted for an acute lacunar stroke in 2016. The personal history included lacunar infarcts in 1997, 2013 and 2015. Her mother had stroke and dementia and her daughter had migraine. Later on, she developed subcortical cognitive impairment.

Cranial MRI showed multiple chronic ischemic lesions in deep white matter, chronic lacunar infarcts (A), an acute lacunar infarction (B) and thalamic-mesencephalic microbleeds (C):

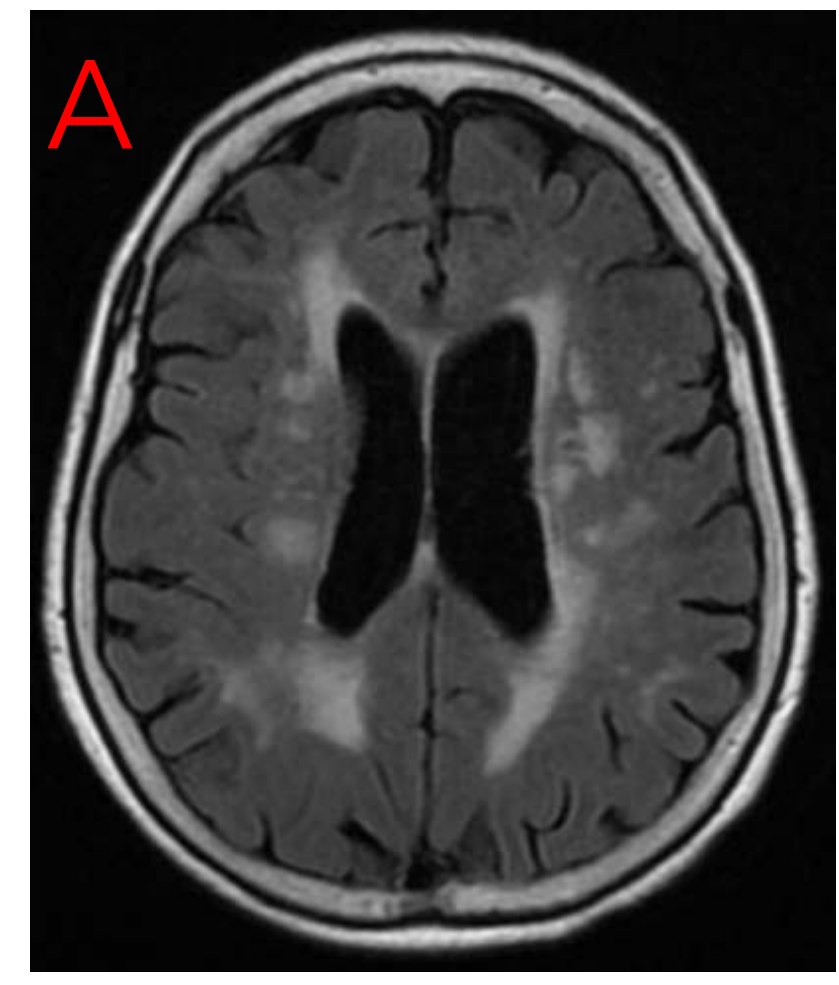

Cranial MRI. FLAIR sequence. Axial view

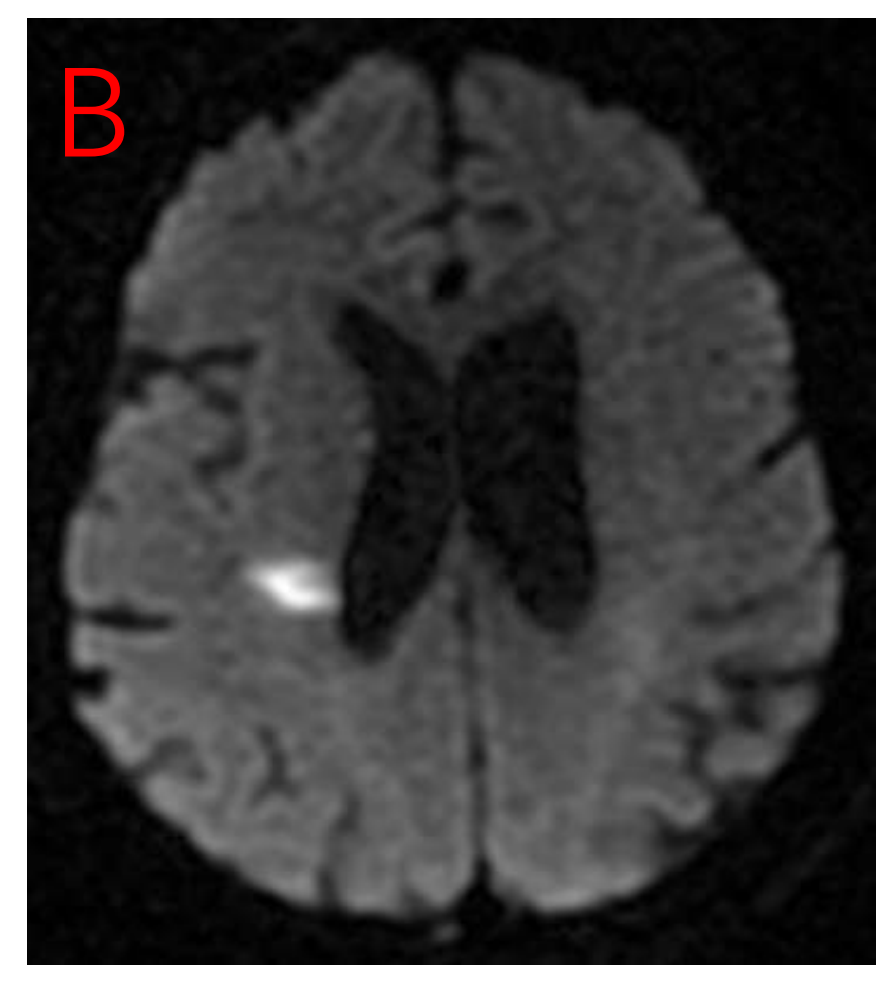

Cranial MRI. DWI sequence. Axial view

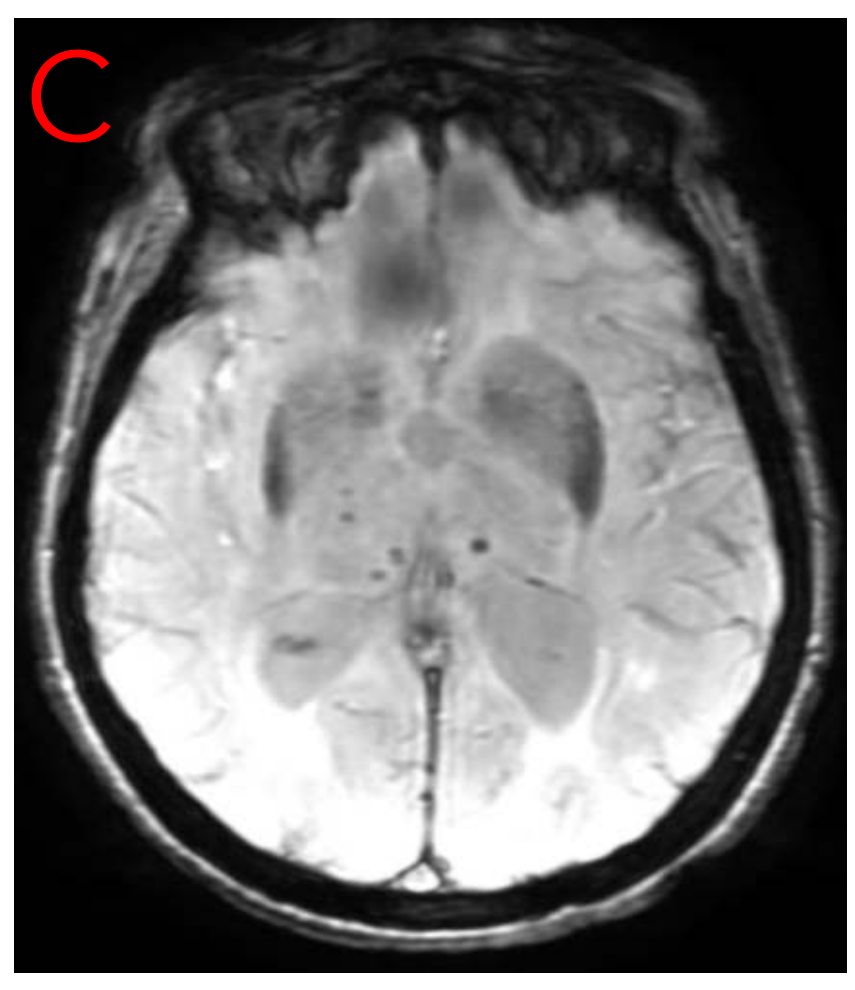

Cranial MRI. T2 gradient echo. Axial view

Case 2: 61-year-old female consulted for a prolonged episode of migraine. She has a family history of migraine. She had no cardiovascular risk factors nor history of stroke. Cranial MRI showed extensive leukoencephalopathy due to small vessel disease:

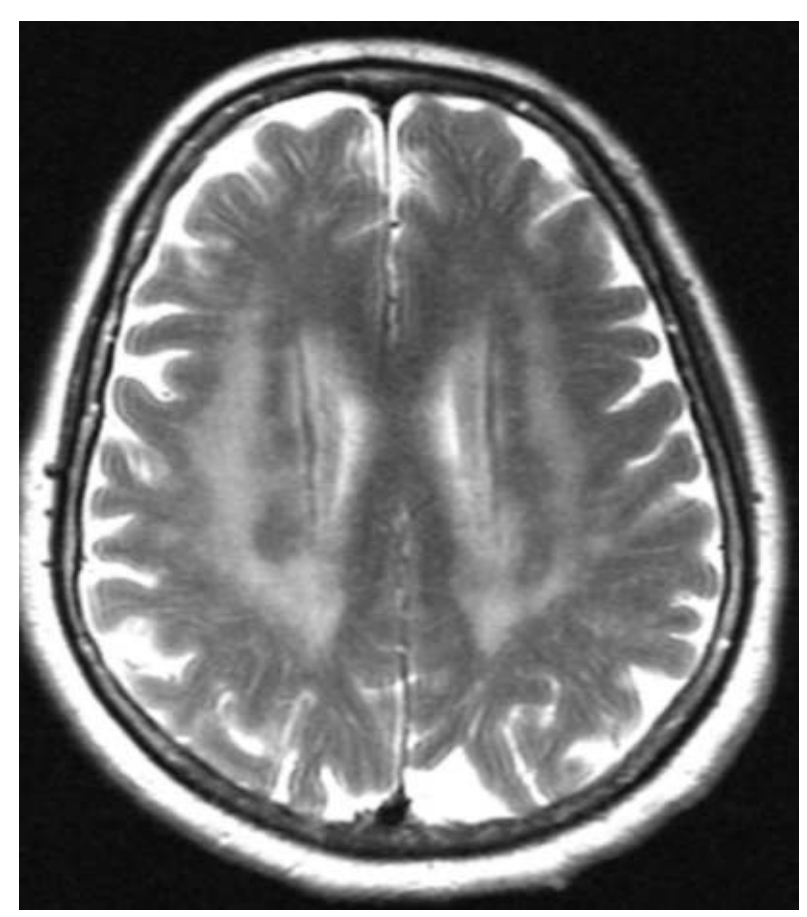

Cranial MRI. T2 sequence. Axial view

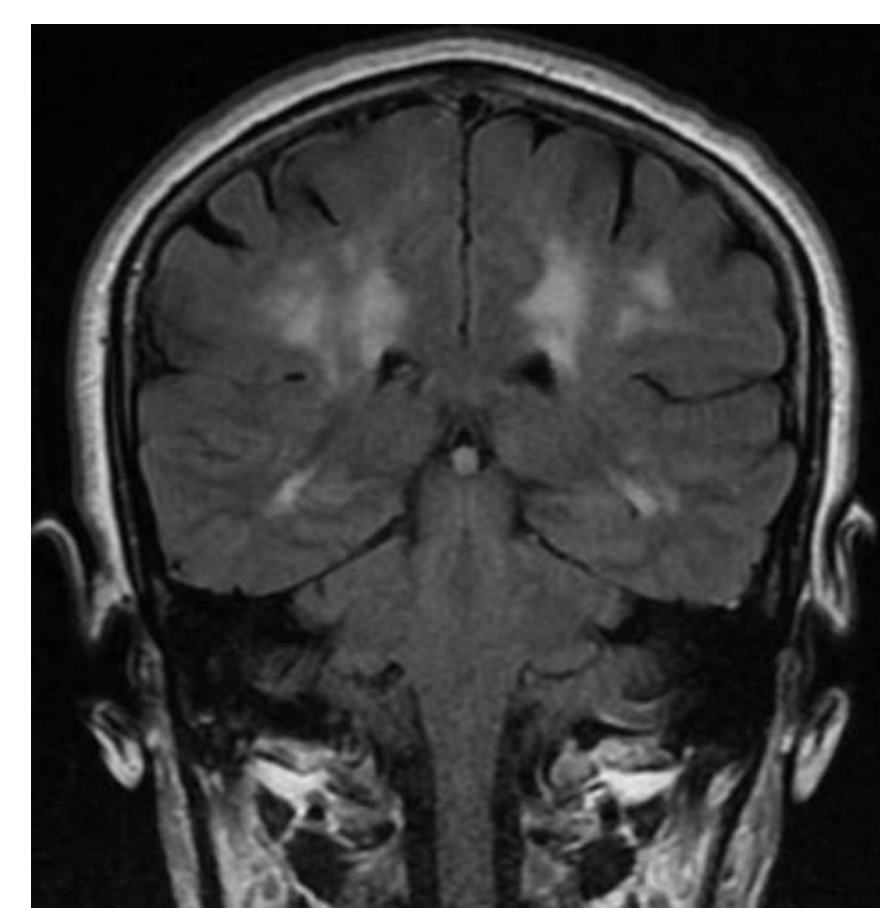

Cranial MRI. T2 FLAIR sequence. Coronal view

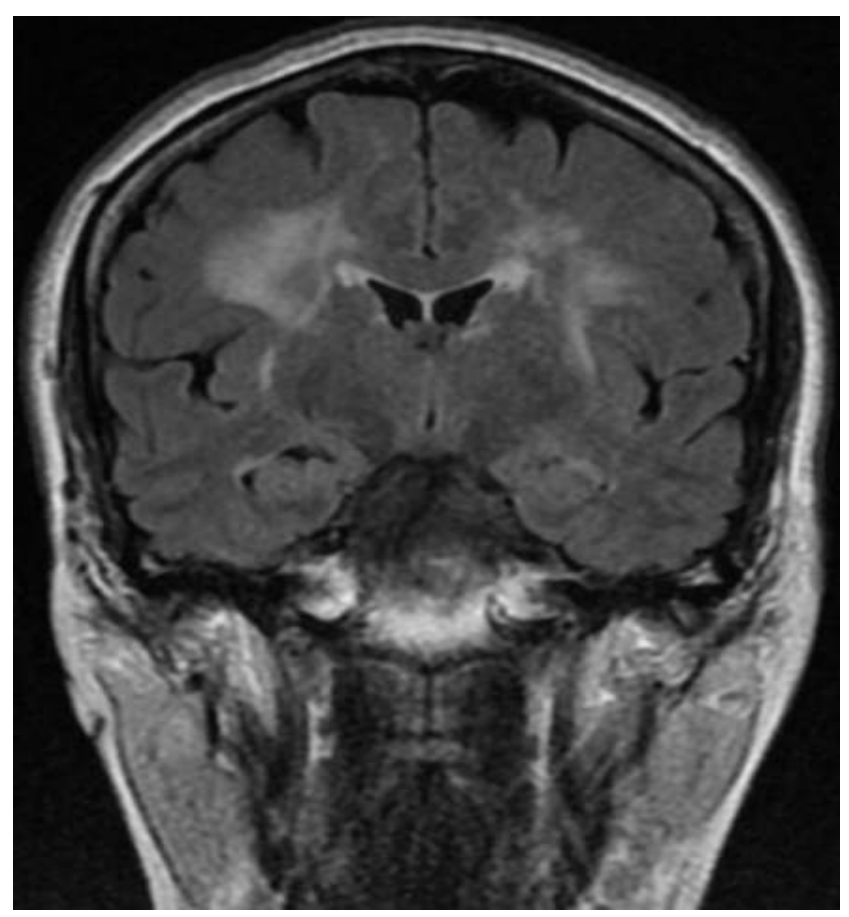

Cranial MRI. T2 FLAIR sequence. Coronal view

\section{RESULTS}

Case 1: The molecular NOTCH 3 study showed an heterozygosity mutation 19th exon [c.3748T> C (p.Cys1250Arg)]. Case 2: The molecular NOTCH 3 study showed an heterozygosity mutation $19^{\text {th }}$ exon [c.3875> G (p.Ser1292Cys)].

\section{CONCLUSIONS}

These mutations have not been described in molecular databases but the analysis with prediction programs indicates that they are probably pathogenic. Since the clinical and radiological data are highly suggestive of CASADIL disease, we have requested a cutaneous biopsy to confirm the diagnosis and to give genetic counseling. 